\title{
Spin as an intrinsic form of top angular momentum
}

\author{
Ivanhoe Pestov ${ }^{1, *}$ \\ ${ }^{1}$ Bogoliubov Laboratory of Theoretical Physics, Joint Institute for Nuclear Research, 141980 Dubna, \\ Moscow Region, Russia
}

\begin{abstract}
In quantum mechanics and particle physics, Spin is considered as an intrinsic form of the quantum orbital angular momentum. The goal of this paper is to demonstrate that in accordance with the creative original idea of Kronig, Uhlenbeck and Goudsmit, Spin can be represented as an intrinsic form of quantum Spherical Top angular momentum. It will be shown that this internal symmetry can be realized on a set of the simplest geometrical quantities, which themselves do not exhibit this emergent property. That is why this phenomenon will be called Emergent Spin. The concept of Spin as an emergent property is more general than the habitual concept of Spin and, hence, it can be interesting in terms of discussion of possible ways to look for a physics beyond the Standard Model. Now, there is no doubt that new physics really exists and we need clear guidance on the best place to look.
\end{abstract}

\section{Introduction}

The Standard Model provides an excellent description of what goes on in the physics of elementary particles. However, we begin to get into trouble when we ask the question of why the Standard Model has the features that it does. Hence, we need to explore a deeper level of nature to find the answer and have a clear guidance to the best place to look for physics beyond the Standard Model. Here we should like to exhibit some physical evidences of the existence of this deeper level and we start from the idea of Spin in the context of the representations of classical mechanics about rotational motion (the concept of "rotating rigid body" also known as the Top).

When Goudsmit and Uhlenbeck proposed the spin hypothesis [1], they had in mind a mechanical picture of the Top. This picture had earlier been considered by Kronig as well. However, it was soon recognized that such an approach could not be realized in a sphere of our immediate experience, connected with movement in space. What does it mean? From classical mechanics it follows that for solution of a certain range of problems it is possible not to consider the internal structure of the object in the question and accept that it is at the same internal state (concept of a "point particle"). With the discovery of quantum mechanics we know that this internal structure exists in the form of a wave field and at this level the internal symmetry plays the same fundamental role as the external symmetry (a transformation of internal symmetry affects functions of the field and does not touch upon the coordinates). Hence, the constructive idea of Kronig, Goudsmit and Uhlenbeck can be considered from a new point of view as internal symmetry, which can be put in correspondence to the quantum

\footnotetext{
*e-mail: pestov@theor.jinr.ru
} 
Spherical Top. The symmetry group of the quantum Spherical Top is well known [2] and it has a transparent geometrical explanation from the four-dimensional point of view [3] (It was detected in [3] that the Spherical Top is a simple geometrical construction strictly defined by a moving point in the four-dimensional Euclidean space). This group is defined by the following dual laws:

$$
\begin{gathered}
{\left[L_{j}, L_{k}\right]=i \varepsilon_{j k l} L_{l}, \quad\left[\widetilde{L}_{j}, \widetilde{L}_{k}\right]=-i \varepsilon_{j k l} \widetilde{L}_{l}, \quad\left[L_{j}, \widetilde{L}_{k}\right]=0 .} \\
L_{1}^{2}+L_{2}^{2}+L_{3}^{2}=\widetilde{L}_{1}^{2}+\widetilde{L}_{1}^{2}+\widetilde{L}_{1}^{2} .
\end{gathered}
$$

An intrinsic form of these relations looks like:

$$
\begin{gathered}
{\left[S_{j}, S_{k}\right]=i \varepsilon_{j k l} S_{l}, \quad S_{1}^{2}+S_{2}^{2}+S_{3}^{2}=s(s+1)=\frac{3}{4},} \\
{\left[\widetilde{S}_{j}, \widetilde{S}_{k}\right]=i \varepsilon_{j k l} \widetilde{S}_{l}, \quad \widetilde{S}_{1}^{2}+\widetilde{S}_{2}^{2}+\widetilde{S}_{3}^{2}=\frac{3}{4}, \quad\left[S_{j}, \widetilde{S}_{k}\right]=0 .}
\end{gathered}
$$

At the fundamental (field-theoretical) level the idea of Spin as intrinsic form of the quantum Spherical Top angular momentum was not realized during the period of the quantum mechanics foundation. And now, in quantum mechanics and particle physics, Spin is considered as an intrinsic form of orbital angular momentum (one additional degree of freedom instead of two, as it should be for the Top; $|+>|-,>\Rightarrow|++>|+,->,|-+>|-,->$ ) with the laws analogous to those of the quantum orbital angular momentum:

$$
\left[S_{j}, S_{k}\right]=i \varepsilon_{j k l} S_{l}, \quad S_{1}^{2}+S_{2}^{2}+S_{3}^{2}=s(s+1)=\frac{3}{4} .
$$

One can put in correspondence to these laws a visual picture of rotation, when the axis of rotation is constant during the motion. In general, it is not the case. It should be noted that the idea of Spin as the intrinsic being of quantum orbital angular momentum was realized in the form of the Dirac equation.

Now our goal is to recognize a deeply hidden structure that can be put in correspondence to equations (3) and (4). It will be shown that the idea of Spin as an intrinsic form of the quantum Spherical Top angular momentum can be realized on a set of the simplest geometrical quantities, which themselves do not exhibit this property. Hence, Spin as intrinsic being of the quantum Spherical Top angular momentum is emergent property and this is the reason to call this phenomenon Emergent Spin. The field which we put in correspondence to Emergent Spin will be called spin field. In connection with the concept of Emergent Spin it should be noted that A.M. Baldin did pay attention to that Reductionism was not the all-inclusive principle of nature and he had the constructive ideas in this direction [4].

\section{Emergent Spin}

Natural geometry is defined by a set of real numbers $R$. A point of this geometry is defined as a 4-tuple of real numbers (in the general case, n-tuple)

$$
x=\left(x^{1}, x^{2}, x^{3}, x^{4}\right) .
$$

More complicated geometries may be constructed on the basis of natural geometry. All points of natural geometry form a reference space $R^{4}$. A set of the simplest (irreducible) geometrical quantities on $R^{4}$ consists of scalar field $a(x)$, covariant vector field $a_{i}(x)$, and antisymmetric covariant tensor fields $a_{i j}(x), a_{i j k}(x)$ and $a_{i j k l}(x)$, which can be arranged as a tuple:

$$
\mathbf{A}=\left(a, a_{i}, a_{i j}, a_{i j k}, a_{i j k l}\right), \quad i, j, k, l=1,2,3,4 .
$$


The set of tuples in question can be considered as linear space of dimension $2^{4}$. With the general definition of linear space we have nothing to do with the preferred dimension of this space. However, it will be shown that from geometrical and group-theoretical points of view dimension $2^{4}$ (in general $2^{n}$ ) is singled out.

Let us exhibit a backbone property that gives an opportunity to interpret $2^{4}$ - tuple (5) as a system. We consider linear transformations (operators) $\hat{\mathrm{T}}: \overline{\mathbf{A}}=\hat{\mathrm{T}} \mathbf{A}$ which are defined by a set of totally antisymmetric tensor fields of type (p,q), $T_{j_{1} \cdots j_{q}}^{i_{1} \cdots i_{p}}, \quad(p, q=0,1,2,3,4)$. We put $\overline{\mathbf{A}}=\left(\bar{a}, \bar{a}_{i}, \bar{a}_{i j}, \bar{a}_{i j k}, \bar{a}_{i j k l}\right), \quad(i, j, k, l=1,2,3,4)$ and have

$$
\begin{gathered}
\bar{a}=T a+T^{m} a_{m}+\frac{1}{2 !} T^{m n} a_{m n}+\frac{1}{3 !} T^{m n p} a_{m n p}+\frac{1}{4 !} T^{m n p q} a_{m n p q}, \\
\bar{a}_{i}=T_{i} a+T_{i}^{m} a_{m}+\frac{1}{2 !} T_{i}^{m n} a_{m n}+\frac{1}{3 !} T_{i}^{m n p} a_{m n p}+\frac{1}{4 !} T_{i}^{m n p q} a_{m n p q} \\
\bar{a}_{i j}=T_{i j} a+T_{i j}^{m} a_{m}+\frac{1}{2 !} T_{i j}^{m n} a_{m n}+\frac{1}{3 !} T_{i j}^{m n p} a_{m n p}+\frac{1}{4 !} T_{i j}^{m n p q} a_{m n p q} \\
\bar{a}_{i j k}=T_{i j k} a+T_{i j k}^{m} a_{m}+\frac{1}{2 !} T_{i j k}^{m n} a_{m n}+\frac{1}{3 !} T_{i j k}^{m n p} a_{m n p}+\frac{1}{4 !} T_{i j k}^{m n p q} a_{m n p q} \\
\bar{a}_{i j k l}=T_{i j k l} a+T_{i j k l}^{m} a_{m}+\frac{1}{2 !} T_{i j k l}^{m n} a_{m n}+\frac{1}{3 !} T_{i j k l}^{m n p} a_{m n p}+\frac{1}{4 !} T_{i j k l}^{m n p q} a_{m n p q} .
\end{gathered}
$$

The identical transformation $\hat{\mathrm{E}}$ is defined by the following natural conditions $E_{j_{1} \cdots j_{q}}^{i_{1} \cdots i_{p}}=$ $0, \quad$ if $\quad p \neq q, \quad E_{j_{1} \cdots j_{p}}^{i_{1} \cdots i_{p}}=\delta_{j_{1} \cdots j_{p}}^{i_{1} \cdots i_{p}}$, where $\delta_{j_{1} \cdots j_{p}}^{i_{1} \cdots i_{p}}$ is the generalized Kronecker delta.

The so defined local internal transformations form the group $G L\left(2^{4}, \mathbf{R}\right)$ which allows one to consider the $2^{4}-$ tuple (5) as a unit. Now it is time to speak about an emergent property. The emergent behavior (or emergent property) can appear when a number of simple entities live in an environment forming unexpected behavior. The number of simple entities is known (see equation (5)). We also know something about the "environment". I mean the linear space of the operators $\hat{\mathrm{T}}$ (defined above). But we know nothing about the hidden properties of this "environment". To detect this underlying structure, we need to introduce one very important element: a positive-definite Riemannian metric field $g_{i j}(x)$, which is a key to realize the laws (3) and (4) and detect the concept of Emergent Spin as well. In this sense, the concept of Emergent Spin has a metric nature.

Indeed, the metric field $g_{i j}(x)$ gives an opportunity to construct a natural general covariant basis in the linear space of the operators $\hat{\mathrm{T}}$ and detect its bipolar structure which means that two dual sets of commuting operators exist. They define the needed basis and can be considered as elements of Lie algebra of two dual subgroups $S$ and $\widetilde{S}$. The transformations of $S$ and $\widetilde{S}$ commute with each other and the spin field $\mathbf{A}$ is the space of the two-valued representation of these dual groups.

To construct the above basis, let us consider the natural algebraic operators $\overline{\mathbf{A}}=\hat{\mathrm{E}}_{\mathbf{v}} \mathbf{A}$ and $\overline{\mathbf{A}}=\hat{\mathrm{I}}_{\mathbf{v}} \mathbf{A}$ defined by the vector field $v^{i}$ as follows:

$$
\begin{gathered}
\hat{\mathrm{E}}_{\mathbf{v}}: \overline{\mathbf{A}}=\hat{\mathrm{E}}_{\mathbf{v}} \mathbf{A}=\left(0, v_{i} a, v_{[i} a_{j]}, v_{[i} a_{j k]}, v_{[i} a_{j k l]}\right), \\
\hat{\mathrm{I}}_{\mathbf{v}}: \overline{\mathbf{A}}=\hat{\mathrm{I}}_{\mathbf{v}} \mathbf{A}=\left(v^{m} a_{m}, v^{m} a_{m i}, v^{m} a_{m i j}, v^{m} a_{m i j k}, 0\right),
\end{gathered}
$$

where the square brackets $[\cdots]$ denote the process of alternation and $v_{i}=g_{i j} v^{j}$. For any vector fields $v^{i}$ and $w^{i}$ we have $\hat{\mathrm{I}}_{\mathbf{v}} \hat{\mathrm{E}}_{\mathbf{w}}+\hat{\mathrm{E}}_{\mathbf{w}} \hat{\mathrm{I}}_{\mathbf{v}}=(\mathbf{v} \mid \mathbf{w}) \cdot \hat{\mathrm{E}}$, where $(\mathbf{v} \mid \mathbf{w})=g_{i j} v^{i} w^{j}$ is a positive-definite scalar product. We mention also the evident relations $\hat{\mathrm{E}}_{\mathbf{v}} \hat{\mathrm{E}}_{\mathbf{w}}+\hat{\mathrm{E}}_{\mathbf{w}} \hat{\mathrm{E}}_{\mathbf{v}}=0, \quad \hat{\mathrm{I}}_{\mathbf{v}} \hat{\mathrm{I}}_{\mathbf{w}}+\hat{\mathrm{I}}_{\mathbf{w}} \hat{\mathrm{I}}_{\mathbf{v}}=0$. To complete, let us introduce the numerical diagonal operator $\hat{Z}$ that is defined by the conditions 
$Z_{j_{1} \cdots j_{q}}^{i_{1} \cdots i_{p}}=0, \quad$ if $\quad p \neq q, \quad Z_{j_{1} \cdots j_{p}}^{i_{1} \cdots i_{p}}=(-1)^{p} \delta_{j_{1} \cdots j_{p}}^{i_{1} \cdots i_{p}}$. From the definition of $\hat{Z}$, we immediately have the following relations: $\hat{\mathrm{E}}_{\mathbf{v}} \hat{\mathrm{Z}}+\hat{\mathrm{Z}} \hat{\mathrm{E}}_{\mathbf{v}}=0, \quad \hat{\mathrm{I}}_{\mathbf{v}} \hat{\mathrm{Z}}+\hat{\mathrm{Z}} \hat{\mathrm{I}}_{\mathbf{v}}=0, \quad \hat{\mathrm{Z}}^{2}=\hat{\mathrm{E}}$.

Now we introduce the fundamental operators:

$$
Q_{\mathbf{v}}=\hat{\mathrm{E}}_{\mathbf{v}}-\hat{\mathrm{I}}_{\mathbf{v}}, \quad \widetilde{Q}_{\mathbf{v}}=\left(\hat{\mathrm{E}}_{\mathbf{v}}+\hat{\mathrm{I}}_{\mathbf{v}}\right) \hat{\mathrm{Z}}
$$

which define the bipolar structure and at the same time the concept of the Emergent Spin (the intrinsic Top).

From the definition of the operators $Q_{\mathbf{v}}$ and $\widetilde{Q}_{\mathbf{v}}$, it follows that

$$
Q_{\mathbf{v}} Q_{\mathbf{w}}+Q_{\mathbf{w}} Q_{\mathbf{v}}=-2(\mathbf{v} \mid \mathbf{w}) \cdot \hat{\mathrm{E}}, \quad \widetilde{Q}_{\mathbf{v}} \widetilde{Q}_{\mathbf{w}}+\widetilde{Q}_{\mathbf{w}} \widetilde{Q}_{\mathbf{v}}=-2(\mathbf{v} \mid \mathbf{w}) \cdot \hat{\mathrm{E}}
$$

and, hence, $Q_{\mathbf{v}}^{2}=\widetilde{Q}_{\mathbf{v}}^{2}=-(\mathbf{v} \mid \mathbf{v}) \cdot \hat{\mathrm{E}}$. Besides, we also deduce one very important relation:

$$
\widetilde{Q}_{\mathbf{v}} Q_{\mathrm{w}}=Q_{\mathrm{w}} \widetilde{Q}_{\mathbf{v}},
$$

that is fulfilled at any vector fields $\mathbf{v}$ and $\mathbf{w}$ and defines the bipolar structure.

Indeed, let us introduce the dual operators

$$
Q_{\mathbf{v} \wedge \mathbf{w}}=\frac{1}{2 !}\left(Q_{\mathbf{v}} Q_{\mathbf{w}}-Q_{\mathbf{w}} Q_{\mathbf{v}}\right), \quad \text { and } \quad \widetilde{Q}_{\mathbf{v} \wedge \mathbf{w}}=\frac{1}{2 !}\left(\widetilde{Q}_{\mathbf{v}} \widetilde{Q}_{\mathbf{w}}-\widetilde{Q}_{\mathbf{w}} \widetilde{Q}_{\mathbf{v}}\right) .
$$

The above formula can be generalized as follows. We take four linear independent vector fields $\mathbf{u}, \mathbf{v}, \mathbf{w}, \mathbf{z}$ and introduce the operators which are defined as an alternated product of the operators $Q_{\mathbf{u}}, Q_{\mathbf{v}}, Q_{\mathbf{w}}, Q_{\mathbf{z}}$ and $\widetilde{Q}_{\mathbf{u}}, \widetilde{Q}_{\mathbf{v}}, \widetilde{Q}_{\mathbf{w}}, \widetilde{Q}_{\mathbf{z}}$. We see that the operators $Q_{\mathbf{u} \wedge \mathbf{v} \cdots \wedge \mathbf{z}}$ and $\widetilde{Q}_{\mathbf{u} \wedge \mathbf{v} \cdots \wedge \mathbf{z}}$ commute with each other and all their possible products put together the needed general covariant basis in the space of linear operators $\hat{T}$. The total number of these operators is equal to $2^{4} \cdot 2^{4}$ (including the identical operator $\hat{\mathrm{E}}$ ). Besides, the operators $Q_{\mathbf{u}}, Q_{\mathbf{v}}, Q_{\mathbf{w}}, Q_{\mathbf{z}}$ and $\widetilde{Q}_{\mathbf{u}}, \widetilde{Q}_{\mathbf{v}}, \widetilde{Q}_{\mathbf{w}}, \widetilde{Q}_{\mathbf{z}}$ and their alternative product can be considered as generators (infinitesimal operators) of the dual groups $S$ and $\widetilde{S}$ mentioned above.

Now let us consider three orthogonal and unit vector fields $\mathbf{u}, \mathbf{v}$ and $\mathbf{w},(\mathbf{u} \mid \mathbf{v})=(\mathbf{u} \mid \mathbf{w})=$ $(\mathbf{v} \mid \mathbf{w})=0,(\mathbf{u} \mid \mathbf{u})=(\mathbf{v} \mid \mathbf{v})=(\mathbf{w} \mid \mathbf{w})=1$ and put

$$
\begin{array}{lll}
S_{1}=\frac{i}{2} Q_{\mathbf{v} \wedge \mathbf{w}}, & S_{2}=\frac{i}{2} Q_{\mathbf{w} \wedge \mathbf{u}}, & S_{3}=\frac{i}{2} Q_{\mathbf{u} \wedge \mathbf{v}}, \\
\widetilde{S}_{1}=\frac{i}{2} \widetilde{Q}_{\mathbf{v} \wedge \mathbf{w}}, & \widetilde{S}_{2}=\frac{i}{2} \widetilde{Q}_{\mathbf{w} \wedge \mathbf{u}}, & \widetilde{S}_{3}=\frac{i}{2} \widetilde{Q}_{\mathbf{u} \wedge \mathbf{v}} .
\end{array}
$$

One can verify that for the operators the above defined relations (3) and (4) are fulfilled and, hence, the internal symmetry in question provides realization of the idea of the intrinsic Top (concept of the Emergent Spin) in the space of spin fields.

We have no opportunity to discuss here equations of the spin field in detail (see [5],[6],[7],[8],[9] ), since it is more important to emphasize here a deep connection between the symmetry group of the quantum Spherical Top and the duality of Time first observed in [10]. We should like to demonstrate that in a certain sense the well-known idea of "rotating rigid body" (also mentioned as the Top) of classical mechanics is as fundamental as the idea of "mass point", i. e. the first concept can be reduced to the second one at the fundamental (field-theoretical) level and dual Time plays a key role in this consideration. First of all, we should like to represent the duality of Time.

A point of the reference space $R^{4}$ (defined above) is a 4-tuple of real numbers $x=$ $\left(x^{1}, x^{2}, x^{3}, x^{4}\right)$. It is clear that the variables $x^{1}, x^{2}, x^{3}, x^{4}$ should be considered on equal footing. Hence, it is unclear how to introduce the so-called space coordinates and time coordinate (a space-time structure) in the framework of reference space alone. To do this, we 
need to define a moment of natural Time. Definition: a moment of natural Time is a number that we put in correspondence to any point of reference space. Hence, a moment of Time is defined by the equation

$$
t=f(x)=f\left(x^{1}, x^{2}, x^{3}, x^{4}\right),
$$

where a scalar potential of natural Time $f(x)$ in the simplest case $\left(g_{i j}=\delta_{i j}\right)$ is a solution for the equations:

$$
\left(\frac{\partial f}{\partial x^{1}}\right)^{2}+\left(\frac{\partial f}{\partial x^{2}}\right)^{2}+\left(\frac{\partial f}{\partial x^{3}}\right)^{2}+\left(\frac{\partial f}{\partial x^{4}}\right)^{2}=1, \quad f\left(\lambda x^{1}, \lambda x^{2}, \lambda x^{3}, \lambda x^{4}\right)=\lambda f\left(x^{1}, x^{2}, x^{3}, x^{4}\right) .
$$

These equations have two solutions:

$$
f(x)=a_{i} x^{i}=a_{1} x^{1}+a_{2} x^{2}+a_{3} x^{3}+a_{4} x^{4},
$$

where $\mathbf{a}=\left(a^{1}, a^{2}, a^{3}, a^{4}\right)$ is a unit constant vector $(\mathbf{a} \mid \mathbf{a})=1$, and

$$
f(x)=\sqrt{\left(x^{1}\right)^{2}+\left(x^{2}\right)^{2}+\left(x^{3}\right)^{2}+\left(x^{4}\right)^{2}} .
$$

All points of the reference space which correspond to the same moment of Time constitute physical space. From the equations

$$
f(x)=a_{i} x^{i}=a_{1} x^{1}+a_{2} x^{2}+a_{3} x^{3}+a_{4} x^{4}=t=\text { constant },
$$

and

$$
f(x)=\sqrt{\left(x^{1}\right)^{2}+\left(x^{2}\right)^{2}+\left(x^{3}\right)^{2}+\left(x^{4}\right)^{2}}=\tau=\text { constant }
$$

we see that in one case the physical space is familiar three-dimensional Euclidean space $E^{3}$ and in another case - the new physical space is three-dimensional Riemannian space of constant positive curvature, i.e. the $3 \mathrm{~d}$-sphere $S^{3}$. The physical (mass) points are to be identified with the points belonging to the three-dimensional Euclidean space $E^{3}$, but the points belonging to the $3 \mathrm{~d}$-sphere $S^{3}$ should be put in correspondence to the Spherical Tops described in [3]. Indeed, the symmetries of Euclidean space can be composed of translations and rotations and the symmetries of the $3 \mathrm{~d}$-sphere $S^{3}$ coincide with symmetries of the Spherical Top (1) and (2). In other words, geometrical points in Euclidean and Riemann spaces have different physical meaning. Thus, from the duality of Time it follows that any known particle can be put in correspondence to a dual particle moving in the dual Time. We see that there are two space-time structures (two different times) on the same reference space. The first space-time structure is well-known as Special Theory of Relativity. The dual Relativity describes the general rotational motion as motion in dual Time. Hence, it is natural to put forward the idea of dual approach to the world of elementary particles which can explain the existence of leptons and quarks, lepton-quark symmetry and confinement (if we identify dual particles with quarks).

Below we exhibit the wave equations describing quantum mechanics of dual particles with the Emergent Spin one half:

$$
\begin{gathered}
\frac{1}{\tau}\left(D+\frac{3}{2}\right) \kappa=\operatorname{div} \mathbf{K}-m \mu \\
\frac{1}{\tau}\left(D+\frac{3}{2}\right) \lambda=\operatorname{div} \mathbf{L}-m v \\
\frac{1}{\tau}\left(D+\frac{3}{2}\right) \mu=\operatorname{div} \mathbf{M}+m \kappa \\
\frac{1}{\tau}\left(D+\frac{3}{2}\right) v=\operatorname{div} \mathbf{N}+m \lambda \\
\frac{1}{\tau}\left(D+\frac{3}{2}\right) \mathbf{K}=-\operatorname{rot} \mathbf{L}+\operatorname{grad} \kappa+m \mathbf{M} \\
\frac{1}{\tau}\left(D+\frac{3}{2}\right) \mathbf{L}=\operatorname{rot} \mathbf{K}+\operatorname{grad} \lambda+m \mathbf{N} \\
\frac{1}{\tau}\left(D+\frac{3}{2}\right) \mathbf{M}=\operatorname{rot} \mathbf{N}+\operatorname{grad} \mu-m \mathbf{K} \\
\frac{1}{\tau}\left(D+\frac{3}{2}\right) \mathbf{N}=-\operatorname{rot} \mathbf{M}+\operatorname{grad} v-m \mathbf{L},
\end{gathered}
$$


where $\tau=\sqrt{\left(x^{1}\right)^{2}+\left(x^{2}\right)^{2}+\left(x^{3}\right)^{2}+\left(x^{4}\right)^{2}}, D=x^{i} \partial_{i}$. The other operators are defined as follows:

$$
(\operatorname{rot} \mathbf{M})^{i}=\frac{1}{\tau} e^{i j k l} x_{j} \partial_{k} M_{l}, \quad(i, j, k, l=1,2,3,4)
$$

where $e^{i j k l}$,is the Levi-Civita tensor normalized as follows $e_{1234}=\sqrt{g}=1$;

$$
(\operatorname{grad} \varphi)_{i}=\triangle_{i} \varphi, \quad \triangle_{i}=\partial_{i}-\frac{x_{i}}{\tau^{2}} D, \quad \operatorname{rot} \operatorname{grad}=0, \quad \operatorname{div} \operatorname{rot}=0
$$

and $(\mathbf{x} \mid \mathbf{K})=(\mathbf{x} \mid \mathbf{L})=(\mathbf{x} \mid \mathbf{M})=(\mathbf{x} \mid \mathbf{N})=0$. The Maxwell equations for dual photons read

$$
\begin{gathered}
\frac{1}{\tau}(D+2) \mathbf{H}=-\operatorname{rot} \mathbf{E}, \quad \frac{1}{\tau}(D+2) \mathbf{E}=\operatorname{rot} \mathbf{H}, \\
(\mathbf{x} \mid \mathbf{E})=(\mathbf{x} \mid \mathbf{H})=0, \quad \operatorname{div} \mathbf{E}=\operatorname{div} \mathbf{H}=0,
\end{gathered}
$$

where

$$
E_{i}=\frac{x^{k}}{\tau} F_{i k}, \quad H_{i}=\frac{x^{k}}{\tau} \stackrel{*}{F}_{i k}, \quad F_{i j}=\partial_{i} A_{j}-\partial_{j} A_{i}, \quad \stackrel{*}{F}_{i j}=\frac{1}{2} e_{i j k l} F^{k l} .
$$

It is natural to put forward conjecture that equations (7),(8) and (9),(10) are a key to discover the internal structure of hadrons and nuclei.

\section{Conclusion}

Since searches for physics beyond the Standard Model have not been successful so far, it has sense to consider new approaches to fundamental physics as a whole. Spin is already a great unifying principle in theoretical physics but its potential is far from being exhausted since the concept of Emergent Spin, as it is demonstrated above, provides new concepts and equations for the physics of elementary particles. Equations (7) and (8) are the result of a shift from the concepts and equations related with the idea of a "rotating rigid body" of classical mechanics to the corresponding natural concepts and equations at the fundamental (field-theoretical) level.

At the present time, quantum chromodynamics has no alternative, but in the framework of this theory we have no answer to the set of principle questions. To explain the confinement mechanism is a very important but still unresolved problem in particle physics. Hence, new approaches and concepts are desirable in any case. From this point of view our suggestion to consider leptons on the ground of the one space-time structure and connect quarks (dual particles) with the dual space-time structure on the same four-dimension Euclidean space looks like quite timely. We put forward a conjecture that this beautiful duality is adequate to the nature of things. The problem of time and everything connected with this topic are up to now significant. The results obtained are significant because they give a simple and evident explanation of lepton-quark symmetry, quark confinement and baryon number conservation. From the point of view of the confinement and the baryon number conservation, it simply means that the quarks (dual particles) cannot change their space-time structure because they are doomed for the eternal natural rotation. At last, we should like to stress that the dual space-time structure is an evident guide in the attempts to find Lorentz violation (I mean a research program on this topic proposed by Alan Kostelesky). 


\section{References}

[1] G.E. Uhlenbeck and S.A. Goudsmit, Nature 117(2938), 264-265 (1926)

[2] L.C. Biedenharn and J.D. Louck, Angular Momentum in Quantum Physics (AddisonWesley Publishing Company, Inc., 1981) 63-65

[3] I.B. Pestov, PoS Baldin ISHEPP XXII, 023 (2015)

[4] A.M. Baldin, Physics of Particles and Nuclear 31(7A), 71-79 (2000)

[5] I.B. Pestov, Theor. and Math. Phys. 34(1), 30-36 (1978)

[6] I.B. Pestov, Physics of Atomic Nuclei, 74(7), 1055-1061 (2011)

[7] I.B. Pestov, J.Phys.Math. 6(135), 1-6 (2015). doi:10.4172/2090-0902.1000135

[8] I.B. Pestov, Eur.Phys.J. Web of Conf. 138, 08008 (2017). doi: 10.1051/epjconf $/ 201713808008$

[9] I.B. Pestov. J.Phys: Conf.Series 938, 012034 (2017). doi: 10.1088/17426596/938/1/012034

[10] I.B. Pestov, Horizons in World Physics 248, Chapter 1 (2005). JINR Preprint E2-200893 